\title{
Erratum: Modeling semiflexible polymer networks
}

\section{[Rev. Mod. Phys. 86, 995 (2014)]}

C. P. Broedersz and F. C. MacKintosh

(published 15 July 2016)

DOI: 10.1103/RevModPhys.88.039903

There is a mistake in Eq. (17). It should be replaced by

$$
\epsilon \equiv \frac{\delta \ell}{\langle\Delta \ell\rangle_{0}}=1-3 \frac{\pi \sqrt{\phi} \operatorname{coth}(\pi \sqrt{\phi})-1}{\pi^{2} \phi} .
$$

